\title{
Comparative Study of the Molecular Beam Epitaxial Growth of InAs/GaSb Superlattices on GaAs and GaSb Substrates
}

\author{
D. Benyahia ${ }^{a, *}$, Ł. Kubiszyn ${ }^{b}$, K. Michalczewski ${ }^{a}$, A. KęBŁOWski ${ }^{b}, \mathrm{P}$ Martyniuk $^{a}$, \\ J. Piotrowski ${ }^{b}$ AND A. RogAlski ${ }^{a}$ \\ ${ }^{a}$ Institute of Applied Physics, Military University of Technology, S. Kaliskiego 2, 00-908 Warsaw, Poland \\ ${ }^{b}$ Vigo System S.A., Poznańska 129/133, 05-850 Ożarów Mazowiecki, Poland
}

\begin{abstract}
Short period type-II $10 \mathrm{ML}$ InAs/10 ML GaSb superlattices epilayers $\left(\lambda_{\text {cut }- \text { off }}=5.4 \mu \mathrm{m}\right)$ have been grown on near lattice matched GaSb (001) substrate and on lattice mismatched GaAs (001) substrate, by molecular beam epitaxy system. In the case of growing on GaAs substrate, GaSb buffer layer was grown in order to reduce the lattice mismatch of $7.5 \%$ between GaAs substrate and InAs/GaSb superlattices. X-ray diffraction characterization shows a good crystalline quality for both samples, with a full width at half maximum of 190 arcsec and 156 arcsec for the zeroth-order peak of the superlattice grown on GaAs and on GaSb substrate, respectively. The Nomarski microscopy revealed a shiny surface for both samples with a root main square of surface roughness of $9 \mathrm{~nm}$ and $11 \mathrm{~nm}$ on the case of growing on GaSb and GaAs substrate, respectively.
\end{abstract}

DOI: 10.12693/APhysPolA.132.322

PACS/topics: 81.15.Hi, 68.65.Cd, 81.05.Ea, 61.05.cp

\section{Introduction}

Infrared (IR) detectors are prominent for several applications ranging from terrestrial defence ones to the commercial ones [1]. Recently, type-II InAs/GaSb strained layer superlattices (SLSs) are considered as a viable alternative to mercury cadmium telluride and quantum well infrared technologies for manufacturing highperformance IR detectors [2,3]. Having been proposed by Sai-Halasz, Tsu and Esaki in 1977 [4], InAs/GaSb superlattices are predicted to offer higher operation temperature, lower dark current, and good spatial uniformity [5]. Moreover, the electronic band structure can be controlled via the variation of the thickness of the constituent layers [6].

The InAs/GaSb SLSs heterostructure exhibits an unique type-II "misaligned" or type-III band alignment where the minimum of InAs conduction layer is situated $140 \mathrm{meV}$ under the top of GaSb valence band [4]. Thus, the fundamental electron-hole transition between the conduction and the heavy hole subbands is controlled by the thickness of each constituent layer. The main advantage of this band alignment is the possibility to suppress the Auger recombination rate due to the large splitting between heavy holes and light holes valence subbands [7]. In addition, the large effective masses of electrons and holes $\left(\approx 0.04 m_{0}\right)$ associated with the InAs/GaSb SLSs should significantly reduce the band-to-band tunneling current [8]. The suppression of the Auger mechanisms and the reducing of tunneling current decrease the dark current, which allows the high temperature operation.

InAs/GaSb SLSs are conventionally grown on lattice matched GaSb substrates to have a low density of thread-

*corresponding author; e-mail: djalal.benyahia@wat.edu.pl ing dislocations. Unfortunately, GaSb substrates are expensive and available on small size less than 3 inch in diameter, which limits the fabrication of large format SLS focal plane arrays (FPAs). Moreover, these substrates are not "epi-ready" and they are with macroscopic defects existing on the growth surface. Furthermore, GaSb substrates exhibit a high absorption coefficient of $\approx 100 \mathrm{~cm}^{-1}$ for IR radiation above $5 \mu \mathrm{m}$ [9]. GaAs substrates have been proposed as a good alternative for the growth of InAs/GaSb SLSs [10-12]. GaAs substrates have excellent structural, optical, and thermal properties. In addition, these substrates are cost-efficient and readily available as large size "epi-ready" wafers up to 6 inch in diameter. Besides, GaAs substrate has an absorption coefficient almost two orders of magnitude lower than that of GaSb substrate. In addition, they are available on large size up to 6 inch in diameter which is beneficial for the cost lowering in the mass production of IR FPAs. Unfortunately, there is a large lattice mismatch of $7.5 \%$ between GaAs substrate and InAs/GaSb SLSs which leads to high misfit dislocations density. Therefore, it is mandatory to develop growth techniques to accommodate the strain and reduce the dislocations density. Several methods have been proposed, among them low temperature nucleation technique [13] and the interfacial misfit dislocation (IMF) array technique [14].

In this paper, we report on the growth of short period $10 \mathrm{ML}$ InAs/10 ML GaSb SLSs with 25 periods, on GaSb (001) substrate as a reference sample, and on GaAs (001) substrate by molecular beam epitaxy (MBE) system. This superlattice structure is concepted to have a cut-off wavelength of $5.4 \mu \mathrm{m}$. A $1.5 \mu \mathrm{m}$ GaSb buffer layer is grown in the case of growing on GaAs substrate in order to accommodate the strain.

\section{Experiment}

Samples were grown by a RIBER Compact 21-DZ solid source MBE system, on GaSb (001) and on GaAs (001) 
substrates. The system is equipped with standard effusion cells for gallium (Ga) and indium (In), and with valved cracked cells for arsenic (As) and antimony $(\mathrm{Sb})$. $\mathrm{As}_{4}$ and $\mathrm{Sb}_{2}$ have been used. The manipulator thermocouple was used to monitor the substrate temperature.

Firstly, in the case of growing on GaSb (001) substrate, after the deoxidization of the substrate at $565^{\circ} \mathrm{C}$, a $2 \mu \mathrm{m}$-thick GaSb buffer layer was deposited at $500^{\circ} \mathrm{C}$ in order to smooth the surface. Next, 25 periods $10 \mathrm{ML}$ InAs/10 ML GaSb SLSs have been grown at $425^{\circ} \mathrm{C}$. The $\mathrm{As} / \mathrm{In}$ and $\mathrm{Sb} / \mathrm{Ga}$ flux ratio was 10.6 and 4.6 , respectively. The growth rate was $0.33 \mu \mathrm{m} / \mathrm{h}$ and $0.76 \mu \mathrm{m} / \mathrm{h}$ for InAs and GaSb, respectively.

Secondly, in the case of growing on GaAs substrate, the deoxidization was at $625^{\circ} \mathrm{C}$. Later, a $0.6 \mu$ m-thick GaAs buffer layer was deposited at $590^{\circ} \mathrm{C}$ in order to get a smooth surface. Then, a $1.5 \mu \mathrm{m}$-thick GaSb layer was grown at $465^{\circ} \mathrm{C}$ to reduce the large lattice mismatch between GaAs substrate and the SLSs. Growth details of GaSb buffer layer are reported elsewhere [15]. Later, 25 periods $10 \mathrm{ML}$ InAs/10 ML GaSb SLSs were deposited at $395^{\circ} \mathrm{C}$. The V/III flux ratio was 8.3 and 4.6 for InAs and GaSb, respectively. The growth rates were the same as in the case of growing on GaSb substrate. The shutters sequence for the growth of InAs/GaSb SLSs is shown in Fig. 1. The growth was monitored in situ by reflection high-energy electron diffraction system (RHEED).

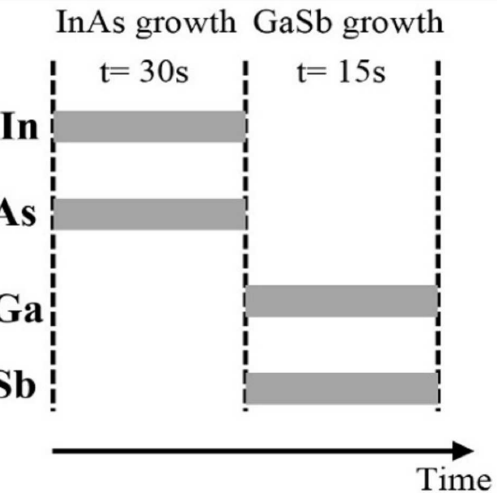

Fig. 1. The shutters sequence for both $\mathrm{InAs} / \mathrm{GaSb} \mathrm{su}-$ perlattices.

The Nomarski optical microscopy was used to assess the surface morphology. On the other hand, the surface roughness has been quantitatively evaluated by means of high-resolution optical profilometry. High-resolution X-ray diffractometer (HRXRD) of PANalytical X'Pert was utilized to assess the structural properties of the InAs/GaSb SLSs. The $\mathrm{Cu} K_{\alpha}$ radiation $(\lambda \approx 1.5406 \AA)$ originating from a line focus was used. The X-ray beam was monochromatized by four bounce, Ge (004) hybrid monochromator. The measurements were performed in $2 \theta-\omega$ direction.

\section{Results and discussion}

The RHEED patterns for GaAs, GaSb, and InAs layers, are illustrated in Fig. 2. The RHEED pattern ex- hibits an ordered $(1 \times 3)$ streaky pattern for GaSb layers, which is typical for this material. On the other hand, the surface reconstruction displays $(2 \times 4)$ diffraction pattern while growing InAs layer. The same pattern was noticed for the growth of GaAs buffer layer. This is indicating a flat surface of these three materials.
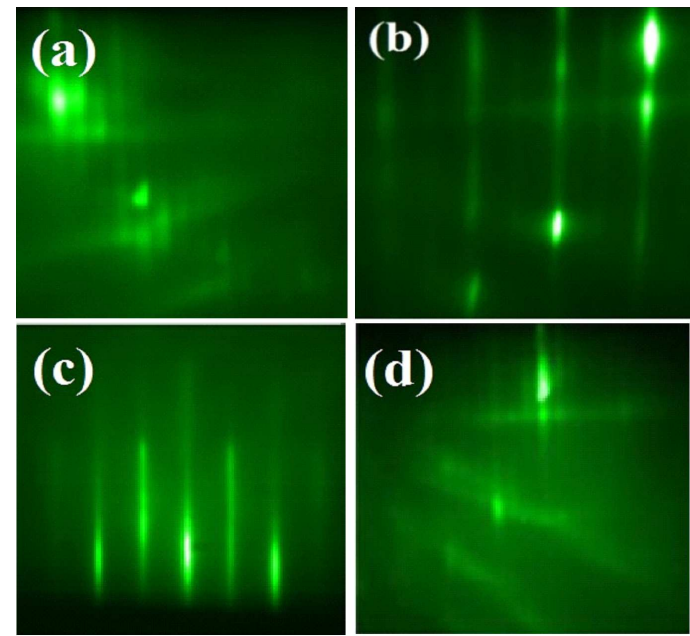

Fig. 2. RHEED patterns (a) $(\times 4),($ b) $(\times 2),(\mathrm{c})(\times 1)$, and $(d)(\times 3)$.

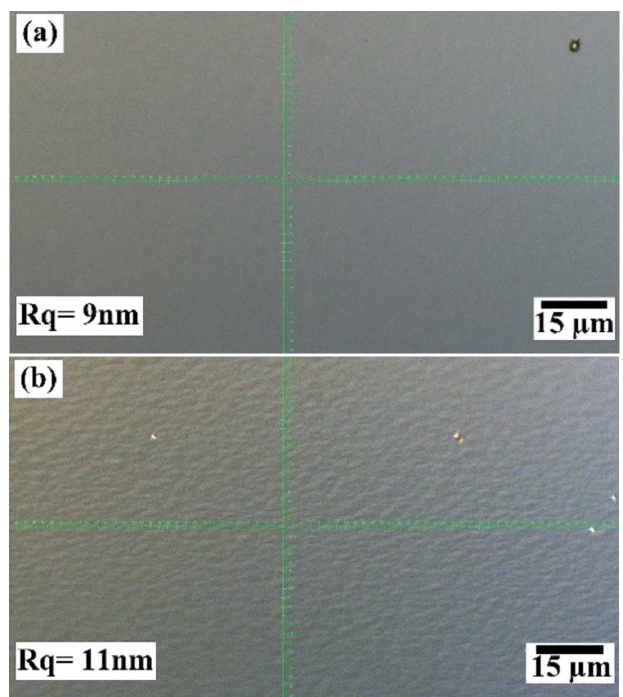

Fig. 3. The Nomarski optical microscopy pictures of InAs/GaSb SLSs (a) grown on GaSb substrate and (b) grown on GaAs substrate.

The surface morphology of the grown InAs/GaSb superlattices is shown in Fig. 3. It can be seen that both samples exhibit a shiny mirror-like surface. A root mean square (RMS) of surface roughness as low as $9 \mathrm{~nm}$ $(11 \mathrm{~nm})$ was obtained in the case of growing on GaSb substrate (GaAs substrate). It is worth noting here that despite the large lattice mismatch between GaAs substrate and the SLSs, the superlattice grown on GaAs substrate exhibits practically the same surface roughness of that grown on lattice matched GaSb substrate. This is 
accomplished using a suitable GaSb buffer layer that reduced the lattice mismatch between the GaAs substrate and the grown superlattices.

Figure 4 presents the high-resolution X-ray diffraction pattern around the symmetric 004 reflection of 25 periods $10 \mathrm{ML}$ InAs/10 ML GaSb SLSs grown on GaAs substrate and GaSb substrate. Figure 4a exhibits one diffraction peak originating from the GaSb substrate and GaSb buffer layer, in addition to well-resolved satellite peaks up to the third order, indicated by " 0 ", " \pm 1 ", etc. This is the evidence of the good crystalline quality and the high reproducibility of the InAs/GaSb SLSs. The full-width at half-maximum (FWHM) of the zeroth-order peak is equal to 156 arcsec. In Fig. $4 \mathrm{~b}$, the $2 \theta-\omega$ scan shows two diffraction peaks, at the position $2 \theta=66.07^{\circ}$ and $2 \theta=60.73^{\circ}$, corresponding to GaAs substrate and GaSb buffer layer, respectively. In addition, there are well-resolved satellite peaks with an order up to three, indicating the high structural quality despite the high lattice mismatch. The FWHM of the zeroth-order peak is 194 arcsec. From the interval between the satellite peaks in Fig. 4, the superlattice periodicity of that grown on GaSb substrate and on GaAs substrate was found to be $52.4 \pm 0.2 \AA$, and $55.3 \pm 0.3 \AA$, respectively, which are less than the expected period of $60.7 \AA$. The residual lattice mismatch $(\Delta a / a)$ of the SLSs grown on GaSb and GaAs substrates is $5.4 \times 10^{-3}$ and $1.7 \times 10^{-2}$, respectively. The low FWHM of the zeroth-order peak is an evidence of the low dislocations density in the superlattice layer [16]. It is important to note that despite the large lattice mismatch between GaAs substrate and InAs/GaSb SLSs compared to that of GaSb substrate, the difference in structural quality between the two sample is very slight.
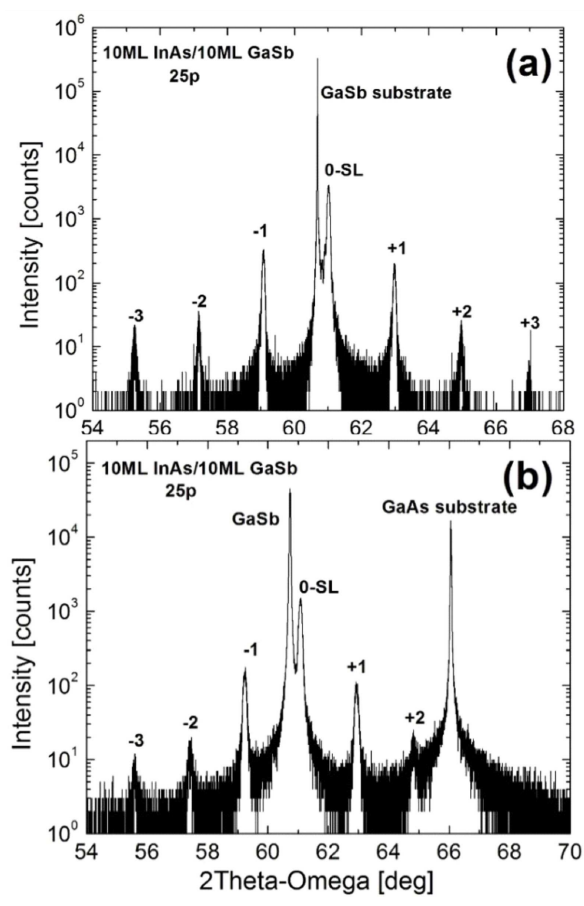

Fig. 4. Experimental $2 \theta-\omega$ symmetric (004) diffraction profile of InAs/GaSb SLSs grown (a) on GaSb substrate and (b) on GaAs substrate.

\section{Summary and conclusions}

The structural properties of short period type-II InAs/GaSb SLSs grown on GaAs and GaSb substrates have been investigated. Good superlattices quality was obtained either on GaSb or GaSb-buffered GaAs substrates. The growth of GaSb buffer layer on GaAs substrate prior to the growth of the SLSs was beneficial for the SLS quality. The large lattice mismatch between the GaAs substrate and InAs/GaSb SLSs, comparable crystalline quality and surface roughness to that of SLSs grown on GaSb substrate were achieved.

\section{Acknowledgments}

This paper has been completed with the financial support of the Polish National Science Centre: grant no. OPUS/UMO-2015/17/B/ST5/01753 and project OPUS/UMO-2015/19/B/ST7/02200.

\section{References}

[1] A. Rogalski, P. Martyniuk, Infrared Phys. Technol. 48, 39 (2006).

[2] M. Razeghi, B.-M. Nguyen, P.-Y. Delaunay, E.K.W. Huang, S. Abdollahi Pour, P. Manurkar, S. Bogdanov, Proc SPIE 7467, 74670T (2009).

[3] E.A. Plis, Adv. Electron. 2014, 1 (2014).

[4] G.A. Sai-Halasz, R. Tsu, L. Esaki, Appl. Phys. Lett. 30, 651 (1977).

[5] D.L. Smith, C. Mailhiot, J. Appl. Phys. 62, 2545 (1987).

[6] H.J. Haugan, F. Szmulowicz, G.J. Brown, K. Mahalingam, Appl. Phys. Lett. 84, 5410 (2004).

[7] C.H. Grein, P.M. Young, M.E. Flatté, H. Ehrenreich, J. Appl. Phys. 78, 7143 (1995).

[8] H. Mohseni, V.I. Litvinov, M. Razeghi, Phys. Rev. B 58, 15378 (1998).

[9] A. Chandola, R. Pino, P.S. Dutta, Semicond. Sci. Technol. 20, 886 (2005).

[10] B.-M. Nguyen, D. Hoffman, E.K.-W. Huang, S. Bogdanov, P.-Y. Delaunay, M. Razeghi, M.Z. Tidrow, Appl. Phys. Lett. 94, 223506 (2009).

[11] B. Tang, Y.-Q. Xu, Z.-Q. Zhou, R.-T. Hao, G.W. Wang, Z.-W. Ren, Z.-C. Niu, Chin. Phys. Lett. 26, 028102 (2009).

[12] X.B. Zhang, J.H. Ryou, R.D. Dupuis, A. Petschke, S. Mou, S.L. Chuang, C. Xu, K.C. Hsieh, Appl. Phys. Lett. 88, 072104 (2006).

[13] E. Michel, H. Mohseni, J.D. Kim, J. Wojkowski, J. Sandven, J. Xu, M. Razeghi, R. Bredthauer, P. Vu, W. Mithcel, M. Ahoujja, Appl. Phys. Lett. 71, 1071 (1997).

[14] S.H. Huang, G. Balakrishnan, A. Khoshakhlagh, A. Jallipalli, L.R. Dawson, D.L. Huffaker, Appl. Phys. Lett. 88, 131911 (2006).

[15] D. Benyahia, Ł. Kubiszyn, K. Michalczewski, A. Kębłowski, P. Martyniuk, J. Piotrowski, A. Rogalski, Opto-Electron. Rev. 24, 1 (2016).

[16] J.E. Ayers, J. Cryst. Growth 135, 71 (1994). 